\title{
Digital Signal Processing Chip Implementation for Detection and Analysis of Intracardiac Electrograms
}

\author{
CHIH-MING JAMES CHIANG, JANICE M. JENKINS, \\ and LORENZO A. DICARLO \\ From the Department of Electrical Engineering and Computer Science, College of Engineering, \\ University of Michigan, and The Michigan Heart and Vascular Institute and Cardiac \\ Electrophysiology Laboratory, St. Joseph Mercy Hospital, Ann Arbor, Michigan
}

CHIANG, C.-M.J., ET AL.: Digital Signal Processing Chip Implementation for Detection and Analysis of Intracardiac Electrograms. The adoption of digital signal processing (DSP) microchips for detection and analysis of electrocardiographic signals offers a means for increased computational speed and the opportunity for design of customized architecture to address real-time requirements. A system using the Motorola 56001 DSP chip has been designed to realize cycle-by-cycle detection (triggering) and waveform analysis using a time-domain template matching technique, correlation waveform analysis (CWA). The system digitally samples an electrocardiographic signal at $1000 \mathrm{~Hz}$, incorporates an adaptive trigger for detection of cardiac events, and classifies each waveform as normal or abnormal. Ten paired sets of single-chamber bipolar intracardiac electrograms (1-500 Hz) were processed with each pair containing a sinus rhythm (SR) passage and a corresponding arrhythmia segment from the same patient. Four of ten paired sets contained intraatrial electrograms that exhibited retrograde atrial conduction during ventricular pacing; the remaining six paired sets of intraventricular electrograms consisted of either ventricular tachycardia (4) or paced ventricular rhythm (2). Of 2,978 depolarizations in the test set, the adaptive trigger failed to detect 6 (99.8\% detection sensitivity) and had 11 false triggers (99.6\% specificity). Using patient dependent thresholds for CWA to classify waveforms, the program correctly identified 1,175 of 1,197 (98.2\% specificity) sinus rhythm depolarizations and 1,771 of 1,781 (99.4\% sensitivity) abnormal depolarizations. From the results, the algorithm appears to hold potential for applications such as realtime monitoring of electrophysiology studies or detection and classification of tachycardias in implantable antitachycardia devices. (PACE 1994; 17:1373-1379)

arrhythmia, intracardiac electrogram, antitachycardia device, implantable defibrillator

\section{Introduction}

Morphological analysis of cardiac waveforms has long been a staple in computerized electrocardiography. Early simplistic measures (duration,

This work was partially supported by National Science Foundation Grants BCS-8909042 and EID-9023514.

Address for reprints: Chih-ming J. Chiang, Ph.D., Telectronics Pacing Systems, 7400 S. Tucson Way, Englewood, CO 80112. Fax: (303) 799-2213.

Received June 25, 1993; revision January 10, 1994; accepted February 2, 1994. amplitude, area under the curve) were advanced for diagnostic electrocardiograms (ECGs). ${ }^{1}$ As computer techniques became more sophisticated, better methods were used, particularly in the classification of normal and abnormal depolarizations for such applications as real-time coronary care monitoring and fast-time scanning of ambulatory (Holter) monitoring. The method of choice in these applications converged in the late 1970 s to a pointby-point comparison of each digitized waveform with a previously extracted template of a normal depolarization. ${ }^{2,3}$ This technique used the classic statistical correlation coefficient, ${ }^{4}$ and later be- 
came known as the correlation waveform analysis (CWA).$^{5-7}$ CWA is a robust measure; it is impervious to the DC offset of the ECG and to amplitude variations of the waveforms. More recently, CWA has been applied to morphological analysis of intracardiac electrograms for recognition of abnormal activation recorded by catheter electrodes. ${ }^{5}$ It has been shown to be equally robust in this setting, given that appropriate bandwidths of the recorded signal be observed. ${ }^{6}$ Due to the heavy computational demands of CWA, however, its only realtime application has been limited to coronary care monitoring systems.

One application that would benefit from incorporation of morphological methods are antitachycardia devices such as pacing and nonpacing implantable cardioverter defibrillators. These devices are designed to terminate potentially lifethreatening tachyarrhythmias such as ventricular tachycardia (VT) and ventricular fibrillation (VF) and have been shown to perform well in preventing sudden cardiac death. ${ }^{8}$ However, these devices utilize simple detection schemes based upon rate and yield a high incidence of false delivery of therapy due to the one-channel nature of detection, which frequently confuses supraventricular arrhythmias with VT and VF. It has been suggested that morphological analysis is the key to alleviate this problem. ${ }^{5,6}$ To date, however, the only realtime programs using CWA for intracardiac electrogram waveform analysis ${ }^{9,10}$ are implemented on the PC-based 386 computer (Intel P700, Intel Corp., Beaverton, OR, USA) or coronary care monitoring machines.

The goal of this study was to demonstrate the feasability of implementing CWA on a digital signal processing (DSP) chip (Motorola 56001 DSP, Motorola Communications \& Electronics, Inc., Schaumburg, IL, USA). This would represent a first step towards specialized architecture incorporating CWA that would be utilized in future generation implantable antitachycardia devices.

\section{Methods}

The overall DSP program contains two main subsections, the first a depolarization detection scheme described in the "Adjustable Threshold Trigger" subsection, and the second the CWA described in the CWA subsection.

\section{Adjustable Threshold Trigger}

An accurate triggering mechanism is essential for rate and morphological analysis of intracardiac electrograms. A previously developed multistage software depolarization detector using adjustable thresholds has been shown to be robust in detecting intracardiac electrograms ${ }^{11}$ and is the basis for the trigger implemented on the DSP chip.

The scheme contains a second order IIR Butterworth band-pass filter that suppresses undesired frequency components and eliminates baseline shift. The trigger has an adaptable threshold with exponential time decay to allow for variability in waveform amplitude. A blanking period is imposed to prevent multiple event detection on a single depolarization.

Figure 1 shows a comprehensive diagram of the trigger. The first stage of the filter is a digital band-pass IIR filter $\mathrm{H}(\mathrm{z})$ derived from the bilinear

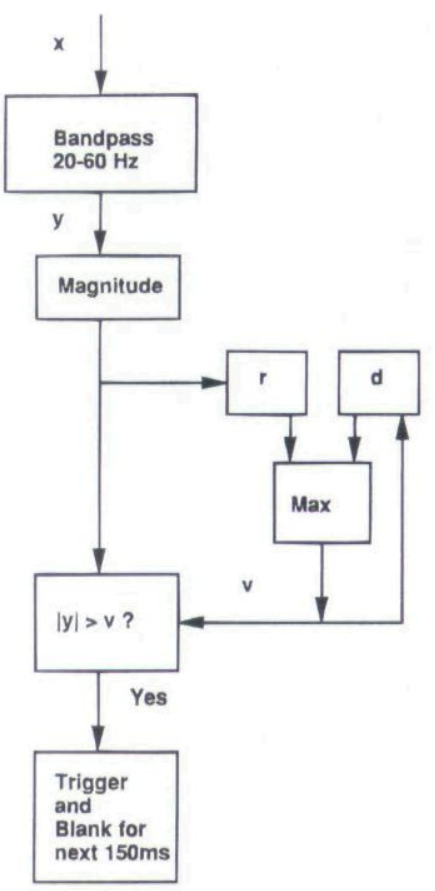

Figure 1. The block diagram for the trigger. $x=$ input, $y=$ output from the bandpass filter, $|y|=$ the magnitude of $y, r=$ fraction to multiply with $|y|, d=$ fraction to multiply with previous $v$ value, $v=$ value from Equation 2, Max $=$ maximum. 
transform of a second order analog filter $\mathrm{H}(\mathrm{s})$ with $s=\left(1-z^{-1}\right) /\left(1+z^{-1}\right)^{11}$ :

$$
\begin{aligned}
& H(s)=\frac{1}{\left(1+s / w_{L}\right)\left(1+w_{H} / s\right)} \\
& H(z)=\frac{a_{0}\left(1-z^{-2}\right)}{1+a_{1} z^{-1}+a_{2} z^{-2}}
\end{aligned}
$$

and $a_{0}, a_{1}, a_{2}$ are derived as follows ${ }^{11}$ :

$$
\begin{aligned}
& a_{0}=\frac{w_{L}}{\left(w_{L}+1\right)\left(w_{H}+1\right)} \\
& a_{1}=\frac{w_{L}-1}{w_{L}+1}+\frac{w_{H}-1}{w_{H}+1} \\
& a_{2}=\frac{\left(w_{L}-1\right)\left(w_{H}-1\right)}{\left(w_{L}+1\right)\left(w_{H}+1\right)}
\end{aligned}
$$

where $w_{\mathrm{L}}, w_{\mathrm{H}}$ are:

$$
\begin{gathered}
w_{L}=\tan \left(\pi f_{L} / f_{s}\right) \\
w_{H}=\tan \left(\pi f_{H} / f_{s}\right)
\end{gathered}
$$

with $f_{\mathrm{L}}$ the lower cut-off frequency, $f_{\mathrm{H}}$ the higher cut-off frequency, and $f_{\mathrm{s}}$ the sampling frequency of the system. The resulting difference equation is:

$$
y_{i}=a_{0}\left(\mathrm{x}_{i}-\mathrm{x}_{i-2}\right)-a_{1} y_{i-1}-a_{2} y_{i-2}
$$

where $y_{i}$ and $x_{i}$ are the ith filter output and input, respectively.

The second stage is a threshold comparison of the magnitude of the filtered signal with an adjustable threshold. The threshold $v$ is the maximum of two components, the first a fraction $(r)$ of the rectified signal and the second a decay factor $(d$ with $d<1$ ) multiplied with the previous threshold. The update function for the threshold is:

$$
v_{i}=\max \left(r\left|y_{i}\right|, d v_{i-1}\right)
$$

The two factors, $r$ and $d$, control the sensitivity of the trigger to allow for detection of waveforms with varying amplitudes. Making $r$ small and $d$ small will increase sensitivity of detection at the expense of increased false triggering probability. The third stage is a blanking interval that prevents multiple triggering during a single depolarization. For this study, values were set as follows: $f_{\mathrm{L}}=20$
$H z, f_{\mathrm{H}}=60 \mathrm{~Hz}, d=2^{-1 / 1000}$, and $r=0.5$ based upon empirical results. ${ }^{11}$

\section{CWA}

The CWA performance measure $\rho$, independent of amplitude fluctuations and baseline changes, yields an output between -1 and +1 where +1 indicates a perfect match. Mathematically, $\rho$ is defined as ${ }^{12}$ :

$$
\rho=\frac{\sum_{i=1}^{i=N}\left(t_{i}-\bar{t}\right)\left(s_{i}-\bar{s}\right)}{\sqrt{\sum_{i=1}^{i=N}\left(t_{i}-\bar{t}\right)^{2}} \sqrt{\sum_{i=1}^{i=N}\left(s_{i}-\bar{s}\right)^{2}}}
$$

where $N=$ the number of points in the template; $t_{\mathrm{i}}=$ the template points; $s_{\mathrm{i}}=$ the signal points to be processed; $\bar{t}=$ the template average; and $\bar{s}=$ the signal average.

To further reduce computation, we eliminate the square root calculation, and rename the metric $\delta=\operatorname{sign}(\rho) \rho^{2}$ where sign is \pm 1 depending on the sign of $\rho$. If normalization of the template points has been calculated (such as by an external programmer) in advance such that $\bar{t}=0$ and $\sum_{k=1}^{k=N} t_{k}^{2}=1$, then the on-line calculation of newly introduced depolarizations becomes ${ }^{12}$ :

$$
\begin{aligned}
\wp & =\operatorname{sign}(\rho) \rho^{2} \\
& =\operatorname{sign}\left(\sum_{i=1}^{i=N}\left(t_{i} s_{i}\right)\right) \frac{\left(\sum_{i=1}^{i=N}\left(t_{i}\right)\left(s_{i}\right)\right)^{2}}{\sum_{i=1}^{i=N} N\left(s_{i}\right)^{2}-\left(\sum_{i=1}^{i=N}\left(s_{i}\right)\right)^{2}}
\end{aligned}
$$

This represents a streamlined version of CWA (using an $N$ point template), which requires $2 N+$ 2 multiplications, 1 division, and $3 N+1$ additions per waveform. This is the equation that is implemented on the DSP chip.

\section{Motorola 56001 System}

The Motorola 56001 DSP chip was designed to perform fast multiply and accumulate operations appropriate for DSP procedures, such as filtering and Fast Fourier Transforms (FFT). ${ }^{13,14}$ The chip is based on a modified Harvard architecture ${ }^{13}$ with a 512 word dynamic random access program memory (DRAM) and two on-chip X and Y data readonly-memory (ROM) as well as data random access memory (RAM) (256 in X and 256 in Y for both RAM and ROM). ${ }^{13,14}$ It has a 24-bit data register 
and two accumulators of 56-bit precision. It executes in fixed point arithmetic with a computational speed of 10.25 million instructions per second (MIPS). ${ }^{13,14}$ The system contains a 16-bit ana$\log$ to digital (A/D) converter with a maximum sampling rate of $44 \mathrm{kHz} \cdot{ }^{13,14}$

The implemented DSP program contains three main components, the analog to digital interface, the triggering section, and the CWA section. Essentially, the algorithm remains in a wait state until an interrupt occurs indicating that data have been received from the A/D. Upon this event, the program stores the data and enters the triggering section. If a depolarization occurs, CWA is activated. CWA is implemented using Equation 4 to reduce computational load. Template values are normalized prior to utilization and a 64 point CWA (64 msec at a $1,000 \mathrm{~Hz}$ sampling rate) was chosen since most intracardiac waveforms fall within this duration and since 64 is a power of $2\left(2^{6}\right)$. To align the template with the waveform being analyzed, the window centered around the trigger for CWA calculation was shifted by $n$ points, with $n$ varying from -10 to +10 , creating 21 separate windows. CWA was performed for each of the windows and the maximum value of the 21 taken as the true CWA performance measure. If this measure was greater than a patient dependent threshold of 0.9 or 0.81 (since signal amplitudes and variabilities were patient dependent, the threshold were chosen individually for each pair of passages), the DSP chip output a positive pulse for a normal depolarization, otherwise it gave a negative pulse indicating an abnormal waveform.

\section{Materials}

Ten pairs of single channel bipolar intracardiac electrograms $(1-500 \mathrm{~Hz})$ from seven patients were processed with each pair consisting of one sinus rhythm (normal) passage and one corresponding arrhythmia segment. Four of the pairs were intraatrial electrograms containing retrograde conduction during ventricular pacing. The other six pairs were intraventricular electrograms containing ventricular tachycardia (4) or ventricular pacing (2).

Data used for analysis were recorded from 6French quadrapolar electrode catheters (USCI Division, C.R. Bard Inc., Billerica, MA, USA) with an interelectrode distance of $1 \mathrm{~cm}$. The recordings were made in the high right atrium and right ventricular apex on FM magnetic tape (Hewlett-Packard Model 3968A, San Diego, CA, USA) at $3 \frac{3}{4}$ inches per second during provocative electrophysiology studies with patients lying supine. For processing, data were digitized through the DSP A/D at $1,000 \mathrm{~Hz}$ and subjected to analysis on the DSP chip.

\section{Results}

Of the 2,978 total depolarizations from the 10 pairs studied, the adaptive trigger missed only 6 (99.8\% sensitivity of depolarization detection) while giving 11 false-positives (99.6\% specificity). Results are shown in Table I. For the performance of CWA with patient dependent thresholds and shifting window calculations (see Motorola 56001 System), the program correctly identified 1,171 of 1,197 (97.8\% specificity) sinus rhythm (normal) depolarizations and 1,771 of 1,781 (99.4\% sensitivity) abnormal depolarizations. The overall result was 2,943 of 2,978 (98.8\%) (Table II).

Figure 2 contains intraventricular electrogram results from patient 4 . The left side of the figure depicts a sinus rhythm passage along with the diagnostic signals and the right side has a VT segment with its corresponding program diagnosis. As can be seen from the sinus rhythm passage, trigger and CWA sections functioned properly with the periodic positive pulse outputs indicating normal waveform diagnosis. For the VT passage, the trigger works equally well and the negative pulse reflects abnormal waveform diagnoses.

Figure 3 contains intraatrial electrogram results from a sinus rhythm passage followed by a ventricular pacing section (yielding retrograde atrial activation) with its corresponding diagnostic pulses. There are no false triggers and all normal depolarizations are indicated in the event marker by positive pulses and abnormal ones by negative pulses. The black blotches represent discontinuities during the taping process and their corresponding results are disregarded when tabulating system performance.

Figure 4 contains an intraatrial electrogram passage that revealed triggering failures. The beginning of the trace shows sinus rhythm followed by ventricular pacing. Note the false-positive triggering in the pacing segment due to a wandering 
Table I.

Result for Real-Time Implementation of the Trigger

\begin{tabular}{|c|c|c|c|c|c|c|c|}
\hline \multirow[b]{2}{*}{ Patient } & \multirow[b]{2}{*}{ Channel } & \multicolumn{3}{|c|}{ Sinus Rhythm } & \multicolumn{3}{|c|}{ Arrhythmia } \\
\hline & & Num & FP & FN & Num & FP & FN \\
\hline 1 & v & 172 & 0 & 0 & 103 & 0 & 0 \\
\hline 2 & v & 87 & 0 & 0 & 86 & 0 & 0 \\
\hline 3 & v & 191 & 2 & 0 & 269 & 0 & 5 \\
\hline 4 & v & 141 & 0 & 0 & 210 & 0 & 0 \\
\hline 5 & A & 146 & 0 & 0 & 249 & 7 & 0 \\
\hline 6 & A & 48 & 0 & 0 & 251 & 0 & 0 \\
\hline 7 & A & 56 & 0 & 0 & 152 & 0 & 0 \\
\hline 8 & v & 50 & 0 & 0 & 134 & 1 & 1 \\
\hline 9 & A & 124 & 0 & 0 & 192 & 0 & 0 \\
\hline 10 & V & 126 & 0 & $\underline{0}$ & 191 & 1 & $\underline{0}$ \\
\hline Total & & $\overline{1,141}$ & 2 & 0 & 1,837 & 9 & $\underline{6}$ \\
\hline Grand Total & & & & & 2,978 & $\overline{11}$ & 6 \\
\hline
\end{tabular}

Num $=$ number; FP $=$ false-positive $; \mathrm{FN}=$ false-negative.

baseline. CWA also failed in one depolarization during sinus rhythm when a normal waveform was declared abnormal.

\section{Discussion and Conclusion}

As shown previously, the DSP system performed well in waveform detection and CWA on intracardiac electrograms. The trigger obtained an accuracy of $99.4 \%$. The few cases of missed triggering $(6 / 2,968)$ resulted from a sequence in which a large amplitude waveform was followed by one of small amplitude, such that the threshold remained too high for the second depolarization to be detected. False-positive triggers occurred due to wandering baseline coupled with small amplitude

Table II.

Result for Real-Time Implementation of CWA

\begin{tabular}{|c|c|c|c|c|c|}
\hline \multirow[b]{2}{*}{ Patient } & \multirow[b]{2}{*}{ Channel } & \multicolumn{2}{|c|}{ Normal } & \multicolumn{2}{|c|}{ Abnormal } \\
\hline & & Num & Wrong & Num & Wrong \\
\hline 1 & V & 170 & 13 & 105 & 0 \\
\hline 2 & V & 84 & 2 & 89 & 0 \\
\hline 3 & V & 191 & 1 & 271 & 6 \\
\hline 4 & V & 137 & 0 & 214 & 0 \\
\hline 5 & A & 146 & 7 & 249 & 0 \\
\hline 6 & A & 70 & 0 & 229 & 0 \\
\hline 7 & A & 59 & 0 & 143 & 0 \\
\hline 8 & V & 50 & 1 & 134 & 0 \\
\hline 9 & A & 164 & 0 & 156 & 4 \\
\hline 10 & V & 126 & $\underline{2}$ & 191 & $\underline{0}$ \\
\hline Total & & $\overline{1,197}$ & $\overline{26}$ & $\overline{1,781}$ & $\underline{10}$ \\
\hline Grand Total & & & & 2,978 & 36 \\
\hline
\end{tabular}

$\mathrm{CWA}=$ correlation waveform analysis; Num $=$ number . 
Ventricular Electrogram

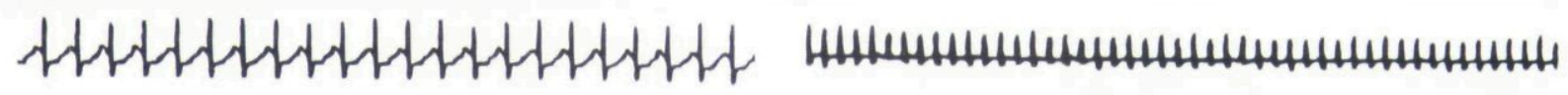

SINUS RHYTHM

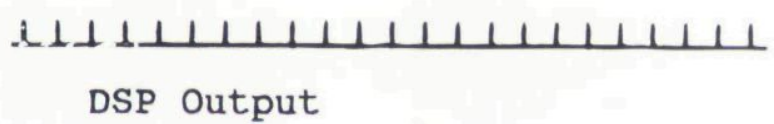

VENTRICULAR TACHYCARDIA

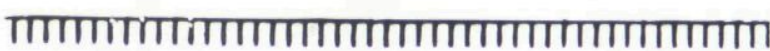

Figure 2. Patient 4 intraventricular electrograms are shown. The left side of the figure contains a sinus rhythm passage with accompanying diagnostic signals and the right side has a ventricular tachycardia passage along with the diagnosis.

signals. The small incidence of these events does not warrant modification of the triggering mechanism. A previous study examining the performance of this depolarization detection scheme showed that the trigger performed well. ${ }^{11}$ The Medtronic PCD (Medtronic, Inc., Minneapolis, $\mathrm{MN}$, USA) detection scheme is also similar to the trigger presented in this study.

For CWA measures, the program was able to distinguish between normal and abnormal waveforms with at least $98 \%$ accuracy using patient dependent thresholds. Most errors were normal waveforms classified as abnormal due to low CWA values and these were mainly due to inaccuracy of the trigger location. The 21-window CWA calculation alleviates most of the problem of inexactness of depolarization detection.

In conclusion, a real-time program has been

Atrial Electrogram

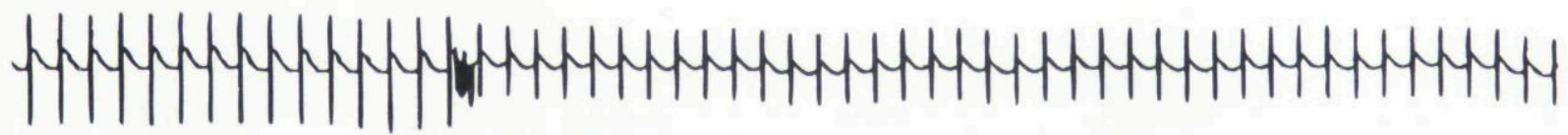

SINUS RHYTHM

VENTRICULAR PACING

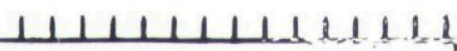

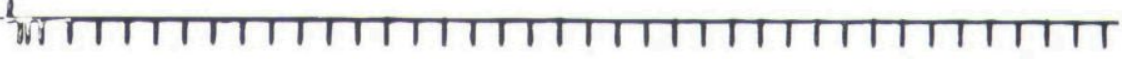

DSP output

Figure 3. Intraatrial electrogram results. Sinus rhythm is followed by pacing. 
Atrial Electrogram

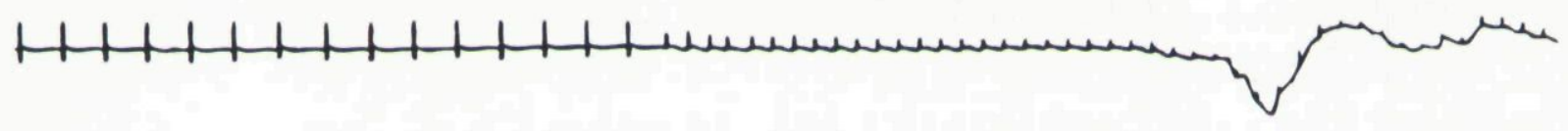

VENTRICULAR PACING

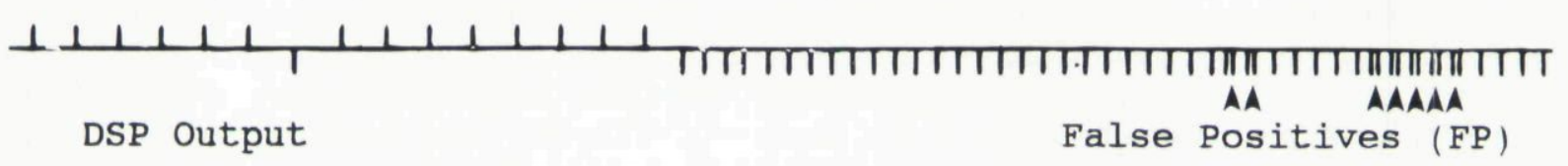

Figure 4. An intraatrial passage with sinus rhythm followed by ventricular pacing. This shows how the wandering baseline corrupts the trigger performance with false-positive (FP) detection of waveforms. Notice also the incorrect digital signal processing (DSP) output of negative pulse on sinus rhythm passage due to low correlation value.

implemented on the Motorola 56001 DSP chip that detects cardiac activation and performs CWA on intracardiac electrograms. The program performs well and represents an important first step towards

\section{References}

1. Jenkins J. Automated electrocardiography and arrhythmia monitoring. Prog Cardiovasc Dis 1983; $25: 367-408$.

2. Arzbaecher R, Biancalana P, Stibolt T, et al. Computer technique for detection of cardiac arrhythmias. (abstract) J Assoc Advance Med Instrument 1971; 5:104.

3. Feldman C, Amazeen P, Klein M, et al. Computer detection of ventricular ectopic beats. Comput Biomed Res 1971; 4:666-674.

4. Kennedy JB, Neville AM. Basic statistical methods for engineers and scientists. Harpers \& Row, Publishers, New York, NY, 1986, pp. 410-411.

5. Lin D, DiCarlo L, Jenkins J. Identification of ventricular tachycardia using intracavitary ventricular electrograms: Analysis of time and frequency domain patterns. PACE 1988; 11:1592-1605.

6. DiCarlo L, Throne R, Jenkins J. A time-domain analysis of intracardiac electrograms for arrhythmia detection. PACE 1991; 14:329-336.

7. Throne R, Jenkins J, Winston S, et al. Discrimination of retrograde from anterograde atrial activation using intracardiac electrogram waveform analysis. PACE 1989; 12:1622-1630.

8. Cohen T, Chien W, Lurie K, et al. Implantable car- miniaturization that would make incorporation of CWA possible in real-time applications such as next-generation implantable antitachycardia devices.

dioverter defibrillator proarrhythmia: Case report and review of the literature. PACE 1991; 14: 1326-1329.

9. Chiang CJ, Jenkins JM, DiCarlo LA. An innovative two-channel rate and morphology method for complex arrhythmia diagnosis in implantable devices. 14th Annual International Conference of the IEEE Engineering in Medicine and Biology Society, 1992;496-497.

10. Chiang CJ, Jenkins JM, DiCarlo LA. Real-time arrhythmia identification from automated analysis of intraatrial and intraventricular electrograms. PACE 1993; 16(1):223-227.

11. McDonald R, Jenkins J, Arzbaecher R, et al. A software trigger for intracardiac waveform detection with automatic threshold adjustment. IEEE Computers in Cardiology, IEEE Press, New York, NY, 1989, pp. 167-170.

12. Throne RD. Analysis of template matching methods for intracardiac electrogram analysis. Ph.D. Thesis, University of Michigan, 1990.

13. Lee E. Programmable DSP Architectures: Part I. IEEE ASSP Magazine 1988; 5(4):4-19.

14. Lee E. Programmable DSP Architectures: Part II. IEEE ASSP Magazine 1989; 6(1):4-14. 
This document is a scanned copy of a printed document. No warranty is given about the accuracy of the copy. Users should refer to the original published version of the material. 